\title{
Long-Term Remission with Ipilimumab/ Nivolumab in Two Patients with Different Soft Tissue Sarcoma Subtypes and No PD-L1 Expression
}

\author{
Maggie Zhou ${ }^{a} \quad N$ am Bui ${ }^{b}$ Marta Lohman ${ }^{b}$ Matt van de Rjin ${ }^{c}$ \\ Gloria Hwang $^{d}$ Kristen Ganjoo ${ }^{b}$ \\ aDepartment of Medicine, Stanford University School of Medicine, Stanford, CA, USA; \\ ${ }^{b}$ Department of Medicine (Oncology), Stanford University School of Medicine, Stanford, CA, \\ USA; 'Department of Pathology, Stanford University, Stanford, CA, USA; ${ }^{\mathrm{d} D e p a r t m e n t}$ of \\ Radiology, Stanford University School of Medicine, Stanford, CA, USA
}

\section{Keywords}

Sarcoma $\cdot$ Ipilimumab $\cdot$ Nivolumab $\cdot$ PD-1 blockade $\cdot$ PD-L1 status

\begin{abstract}
Checkpoint inhibitor therapy has been shown to improve outcomes in multiple solid malignancies; however, data are limited in soft tissue sarcoma. We present two cases of patients with advanced soft tissue sarcoma of different subtypes (dedifferentiated liposarcoma and myxofibrosarcoma) with zero percent PD-L1 expression by immunohistochemistry who were treated with ipilimumab and nivolumab followed by maintenance nivolumab. Both patients had failed multiple lines of systemic treatment and experienced long-term remission after starting ipilimumab and nivolumab. Genetic testing revealed that no genetic mutations were found in common between the two cases. One patient received concurrent cryoablation, which may have sensitized his tumor to immunotherapy. Checkpoint inhibitor therapy may improve outcomes in soft tissue sarcoma regardless of PD-L1 status, especially when combined with cryoablation. Studies are needed to evaluate whether treatment response varies by sarcoma subtype and what molecular markers can be used to guide patient selection.
\end{abstract}




\section{Introduction}

Checkpoint blockade has shown promising efficacy in multiple malignancies [1-5], but limited data exist regarding the efficacy and tolerability in soft tissue sarcoma (STS). The ALLIANCE trial demonstrated the superiority of ipilimumab in combination with nivolumab over single-agent nivolumab in STS [6], with an objective response rate of $15 \%$. However, there are limited data on the efficacy of ipilimumab and nivolumab in real-world clinical practice, and little is known regarding the correlation of treatment effect with PD-L1 status. In this report, we present two cases at our institution of patients with advanced STS of different subtypes (dedifferentiated liposarcoma and myxofibrosarcoma) that had excellent long-term responses to ipilimumab and nivolumab despite negative PD-L1 status.

\section{Case Report}

\section{Case 1}

A 67-year-old Caucasian male had resection of a right axillary dedifferentiated liposarcoma in May 2013 with positive margins followed by adjuvant radiation. In January 2016, he had biopsy-proven local recurrence without metastatic disease. He had stable disease with 8 cycles of doxorubicin and olaratumab followed by 8 cycles of maintenance olaratumab. He had disease progression and was treated with gemcitabine and docetaxel. In February 2018, due to disease progression, he was switched to pazopanib. His course was complicated by soft tissue infection related to the axillary mass and systolic heart failure attributed to anthracycline-related cardiomyopathy in the setting of increased afterload from tyrosine kinase inhibitor (left ventricular ejection fraction 21\%). In April 2018, PET scan showed progression of right axillary mass and diffuse metastatic disease involving the lungs, pleura, and liver. The patient began off-label treatment with ipilimumab $(1 \mathrm{mg} / \mathrm{kg})$ and nivolumab $(3 \mathrm{mg} / \mathrm{kg})$ every 3 weeks for four cycles followed by maintenance nivolumab $(3 \mathrm{mg} / \mathrm{kg})$ every 2 weeks. His cardiac function recovered shortly after starting ipilimumab/nivolumab. The patient received concurrent cryoablation of the right axillary mass in June 2018 (Fig. 1). Subsequent restaging CT scans demonstrated a significant response in the right axillary mass, pulmonary nodules, and liver lesions (Fig. 2). After completion of four cycles of ipilimumab and nivolumab and two cycles of maintenance nivolumab, he developed autoimmune colitis and was treated with prednisone taper for 8 weeks. He developed pneumonia in the setting of immunosuppression that was complicated by bacteremia and atrial fibrillation and experienced an acute decline in cardiac function. After hospital discharge, he continued maintenance nivolumab at $6 \mathrm{mg} /$ kg every 4 weeks. He remained on maintenance nivolumab, with impressive radiographic and clinical response including disappearance of his axillary mass. In December 2018, he passed away from sudden cardiac death.

Case 2

A 66-year-old Asian female had margin negative resection of myxofibrosarcoma of the right calf in March 2012 followed by adjuvant radiation. In May 2013, she had a margin negative resection of a recurrence in her right upper thigh. In August 2015, she had a resection of a recurrence extending to more proximal thigh and pelvis followed by radiation. In August 2016, she had resection of right upper thigh and gluteus minimus sarcoma. She started treatment with pazopanib, which was complicated by severe hypertension requiring multiple anti-hypertensives. She was started on doxorubicin and olaratumab due to disease progression in November 2016. She had initial treatment response but demonstrated disease progression in July 2017, and she was started on ipilimumab (1 mg/kg) and nivolumab (3 mg/kg) every

\section{Karger's}




\section{Case Reports in Oncology}

\begin{tabular}{|c|c|}
\hline Rep Oncol 2021;14: & \\
\hline DOI: 10.1159/000512828 & $\begin{array}{l}\text { (c) } 2021 \text { The Author(s). Published by S. Karger AG, Basel } \\
\text { www.karger.com/cro }\end{array}$ \\
\hline
\end{tabular}

Zhou et al.: Remission with Ipilimumab/Nivolumab in Two Patients without PD-L1 Expression

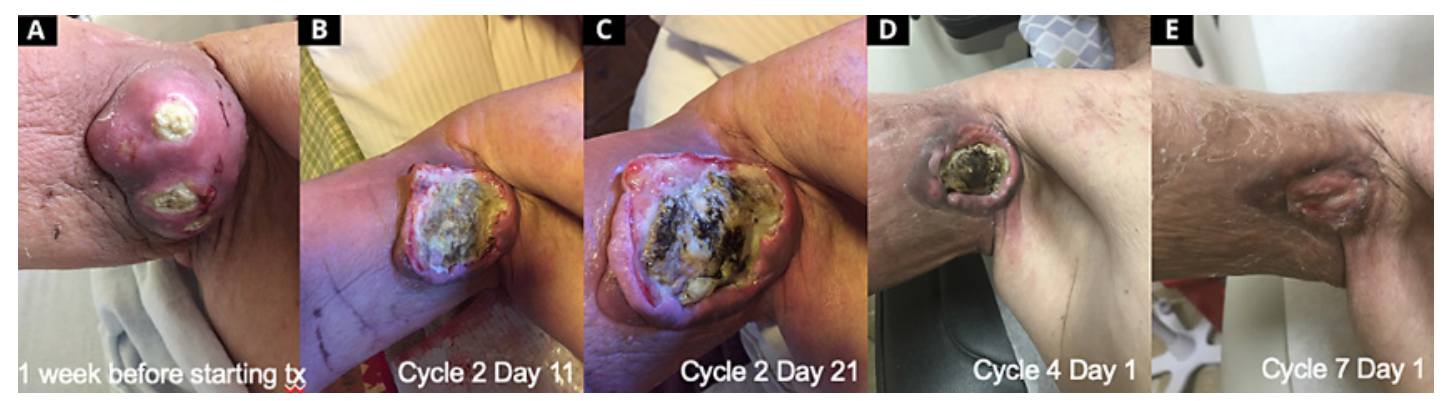

Fig. 1. Response of axillary dedifferentiated liposarcoma to treatment with ipilimumab and nivolumab: 1 week before starting treatment (A), cycle 2 day 11 (B), cycle 2 day 21 (C), cycle 4 day 1 (D), and cycle 7 day 1 (E). The patient received four cycles of induction therapy about 21 days each; cycle 3 was delayed by 2 days, and cycle 4 was delayed by 1 day. After completing induction therapy, the patient received maintenance therapy about every 14 days for cycles 5 and 6, and about every 28 days starting cycle 7 . The patient received concurrent cryoablation on cycle 3 day 20 .

Fig. 2. Baseline and follow-up radiographic imaging of the patient with dedifferentiated liposarcoma treated with ipilimumab and nivolumab followed by maintenance nivolumab therapy, with concurrent cryoablation. Top: cycle 1 day 1 , axillary mass measuring $8.1 \times 5.9 \times 9.8 \mathrm{~cm}$ with SUV max 12.2; middle: cycle 5 day 8 , largely resolved mass with SUV max 4.7; bottom: cycle 10 day 1 , stable or improving posttreatment changes. A yellow arrow indicates the lesions.

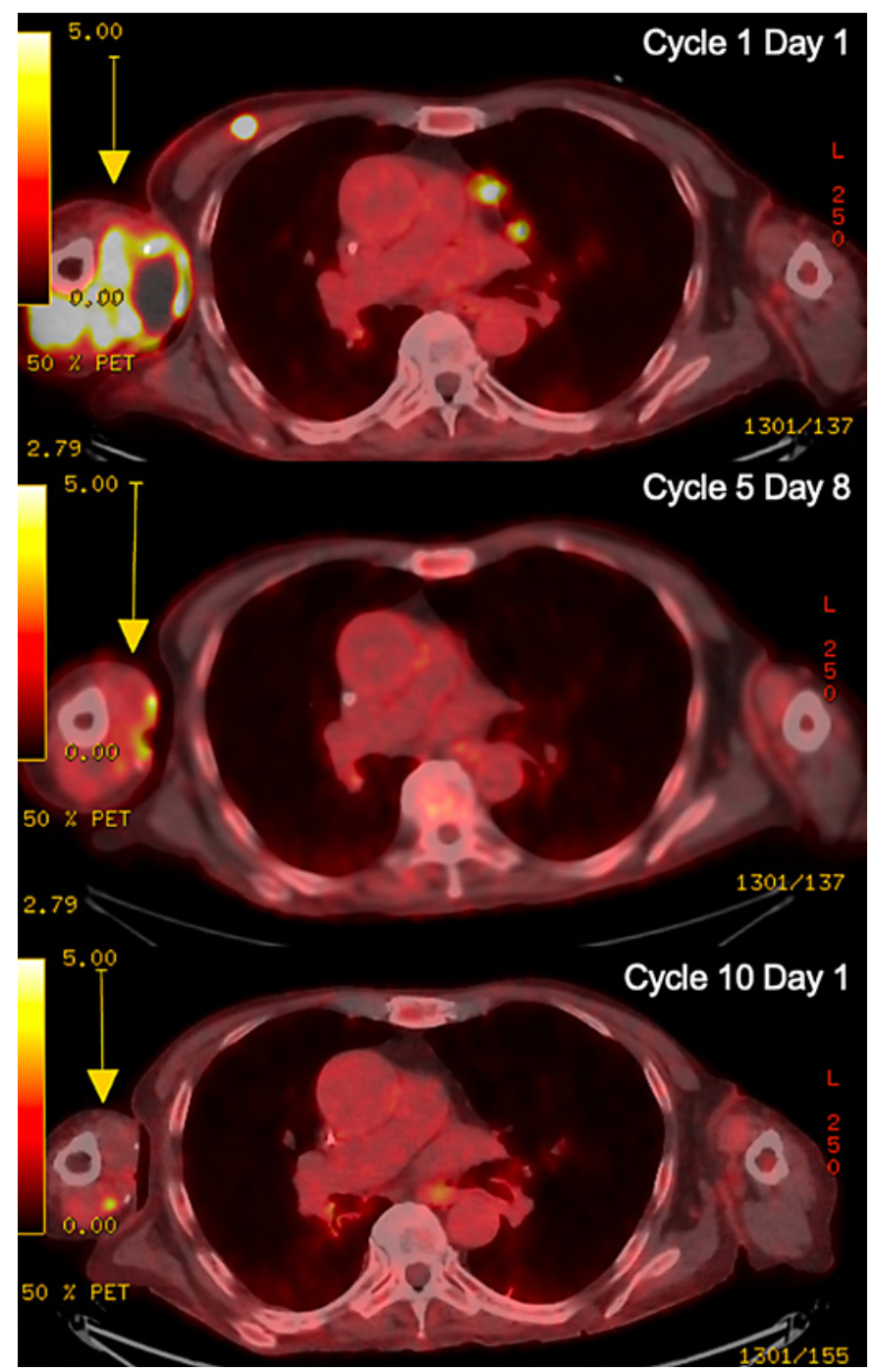


Zhou et al.: Remission with Ipilimumab/Nivolumab in Two Patients without PD-L1 Expression

Fig. 3. Baseline and follow-up radiographic imaging of the patient with myxofibrosarcoma treated with ipilimumab and nivolumab followed by maintenance nivolumab therapy. Top: 1 week before starting treatment; middle: cycle 19 day 14 ; bottom: 21 months after starting treatment.

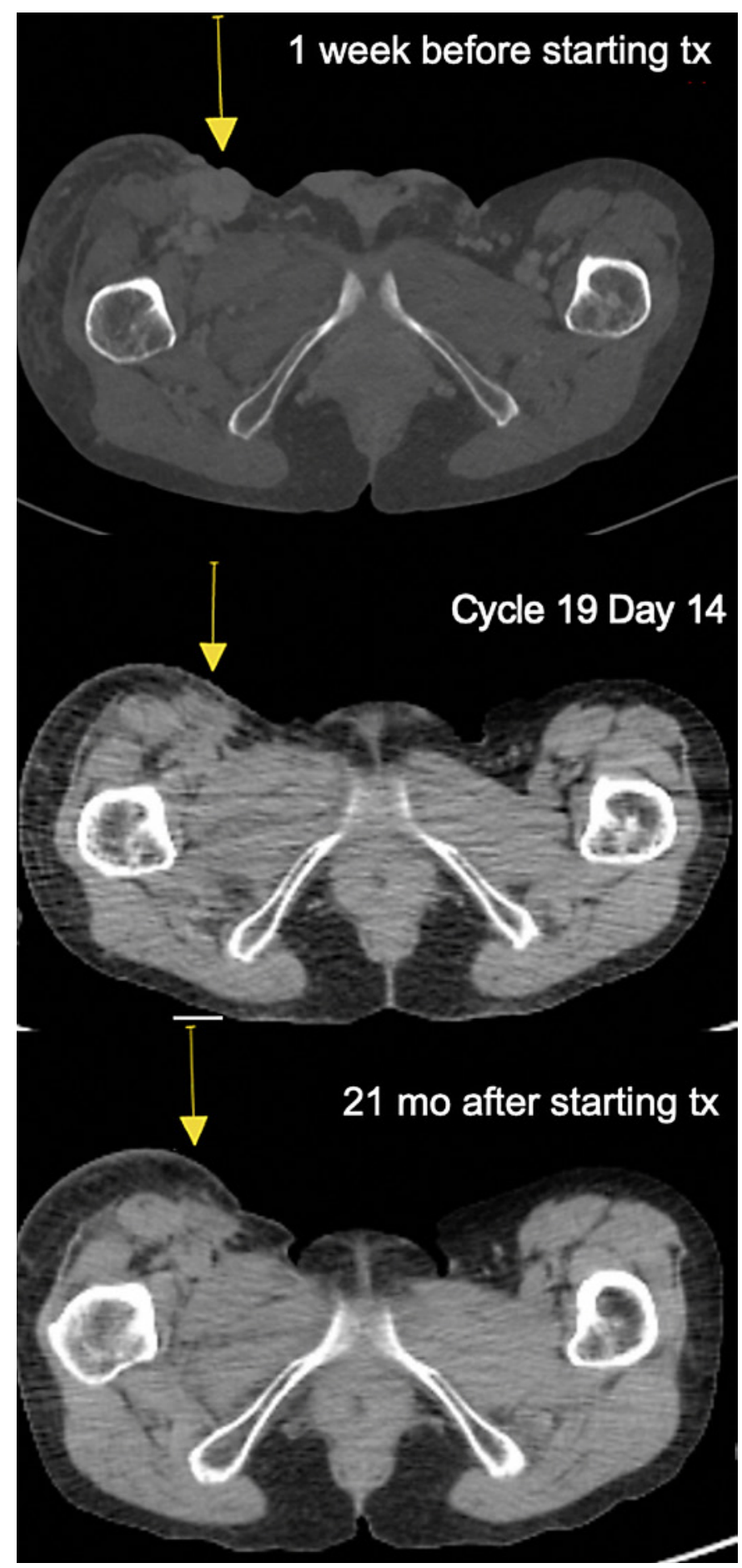

4 weeks for four cycles followed by maintenance nivolumab (6 mg/kg) every 4 weeks. Her subcutaneous nodules decreased in response to treatment (Fig. 3). In December 2017, she was admitted for progressive dyspnea on exertion, orthopnea, and lower extremity edema, with workup confirming new-onset acute systolic heart failure with ejection fraction $29 \%$. Troponin peaked at 0.15 , and coronary angiogram did not reveal any obstructive coronary disease. No delayed enhancement was seen on cardiac MRI. She was evaluated by cardiology, 
and given the lack of myocarditis or scar on MRI and prior significant anthracycline dose, cardiology favored anthracycline effect as etiology of her non-ischemic cardiomyopathy. She underwent diuresis and was started on carvedilol and lisinopril for optimal medical management of heart failure. Given her continued response to nivolumab, it was resumed January 2018 with the plan to continue until progression or unacceptable toxicity. Her cardiac function recovered to baseline by May 2018. As of July 2020, she has received 50 cycles of immunotherapy and remains on maintenance nivolumab with complete response.

\section{Discussion}

We present two cases of patients with advanced STS of different subtypes (dedifferentiated liposarcoma and myxofibrosarcoma) who had excellent long-term responses to ipilimumab and nivolumab despite negative PD-L1 status. The patient with dedifferentiated liposarcoma had an almost complete response in the axillary mass and partial response in the lungs and liver but passed away from congestive heart failure that predated his immunotherapy treatment and was attributed to anthracycline-related cardiomyopathy. The patient with myxofibrosarcoma has had a complete response for over 3 years and remains without disease on treatment. Both patients had failed multiple lines of systemic treatment and experienced long-term remission after starting ipilimumab and nivolumab. Genetic testing (suppl. Table 1; see www.karger.com/doi/10.1159/000512828) revealed no genetic mutations in common between the two cases.

The excellent response to PD-1 blockade despite negative PD-L1 status could be due to numerous mechanisms. Cryoablation has been suggested to sensitize tumors to immunotherapy $[7,8]$. In one proposed mechanism, tumor antigens released after cryoablation are taken up by antigen-presenting cells, which mature and induce tumor-specific T-cell activation and proliferation [9]. PD-1 inhibition prevents these tumor-specific T cells from developing anergy. Cryoablation combined with immunotherapy has shown promise in a pilot study of early-stage breast cancer treated with preoperative percutaneous tumor cryoablation, ipilimumab, or both, followed by mastectomy [10]. Cryoimmunotherapy has also been investigated in a pilot trial in newly diagnosed oligometastatic prostate cancer with wholeprostate cryoablation combined with short-term androgen deprivation and pembrolizumab [11] based on research in prostate cancer murine models [12]. There is an ongoing phase II clinical trial investigating the combination of percutaneous cryoablation and ipilimumab/ nivolumab for advanced STS (NCT04118166).

Similarly, radiotherapy may sensitize tumor to checkpoint blockade. PD-L1 status may turn positive after radiation, which has been shown in non-small cell lung cancer [13]. PD-L1 is regulated by at least two mechanisms in tumors: intrinsic immune resistance and adaptive resistance, the former in responding to oncogenic signaling, the latter in responding to cytokines like IFN-r. It is possible that PD-L1 frequently acts as a marker of adaptive immune resistance in response to engaged $\mathrm{CD} 3+$ and $\mathrm{CD} 8+$ tumor-infiltrating lymphocytes, and represents a negative feedback mechanism rather than a constitutive biomarker [14]. While high PD-L1 expression is associated with greater benefit from immunotherapy, patients with low PD-L1 expression at time of testing have better survival than with standard of care [15, 16].

B cells have also been gaining attention in clinical tumor immunology as a biomarker for response to immunotherapy. Previously, B cells were largely thought to inhibit the inflammatory anti-tumor response as regulatory B cells. Studies in melanoma show tumor-associated B cells also sustain the inflammatory tumor microenvironment through plasmablastlike cells. These plasmablast-like cells can in turn increase PD- $1+\mathrm{T}$ cell activation through

\section{Karger'}


anti-PD-1 blockade, and their frequency predicts response and survival to immune checkpoint blockade $[17,18]$. In STS, enrichment of B cells was a hallmark of response to checkpoint blockade [19].

\section{Conclusion}

In conclusion, immune checkpoint blockade has shown promising efficacy in multiple malignancies [1-5]; however, limited data exist regarding the efficacy and tolerability in STS. Our experience suggests that ipilimumab and nivolumab can provide long-term disease control for patients with advanced STS in real-world clinical practice, irrespective of PD-L1 status.

\section{Statement of Ethics}

Written informed consent was obtained from the deceased patient's next of kin (case 1) and from the patient directly (case 2) for publication of this case report and any accompanying images.

\section{Conflict of Interest Statement}

The authors have no conflicts of interest to disclose.

\section{Funding Sources}

The authors did not receive any funding.

\section{Author Contributions}

M.Z. drafted the manuscript, M.Z., N.B., G.H., and K.G. revised the manuscript; all authors were substantially involved in the conception and design of the case report, as well as in the acquisition, analysis, and interpretation of the data; all authors approved the final version.

\section{References}

1 Herbst RS, Baas P, Kim DW, Felip E, Pérez-Gracia JL, Han JY, et al. Pembrolizumab versus docetaxel for previously treated, PD-L1-positive, advanced non-small-cell lung cancer (KEYNOTE-010): a randomised controlled trial. Lancet. 2016;387(10027):1540-50.

2 Plimack ER, Bellmunt J, Gupta S, Berger R, Chow LQ, Juco J, et al. Safety and activity of pembrolizumab in patients with locally advanced or metastatic urothelial cancer (KEYNOTE-012): a non-randomised, openlabel, phase 1b study. Lancet Oncol. 2017;18(2):212-20.

3 Nghiem PT, Bhatia S, Lipson EJ, Kudchadkar RR, Miller NJ, Annamalai L, et al. PD-1 Blockade with Pembrolizumab in Advanced Merkel-Cell Carcinoma. N Engl J Med. 2016;374(26):2542-52.

4 Chen R, Zinzani PL, Fanale MA, Armand P, Johnson NA, Brice P, et al. Phase II Study of the Efficacy and Safety of Pembrolizumab for Relapsed/Refractory Classic Hodgkin Lymphoma. J Clin Oncol. 2017;35(19):2125-32.

5 Hamid O, Robert C, Daud A, Hodi FS, Hwu WJ, Kefford R, et al. Safety and tumor responses with lambrolizumab (anti-PD-1) in melanoma. N Engl J Med. 2013;369(2):134-44.

\section{Karger'₹}




\section{Case Reports in Oncology}

\begin{tabular}{l|l}
\hline Case Rep Oncol 2021;14:459-465 \\
\hline DOI: 10.1159/000512828 & $\begin{array}{l}\text { @ 2021 The Author(s). Published by S. Karger AG, Basel } \\
\text { www.karger.com/cro }\end{array}$ \\
\hline
\end{tabular}

Zhou et al.: Remission with Ipilimumab/Nivolumab in Two Patients without PD-L1 Expression

6 D'Angelo SP, Mahoney MR, Van Tine BA, Atkins J, Milhem MM, Jahagirdar BN, et al. Nivolumab with or without ipilimumab treatment for metastatic sarcoma (Alliance A091401): two open-label, non-comparative, randomised, phase 2 trials. Lancet Oncol. 2018;19(3):416-26.

7 Katzman D, Wu S, Sterman DH. Immunological Aspects of Cryoablation of Non-Small Cell Lung Cancer: A Comprehensive Review. J Thorac Oncol. 2018;13(5):624-35.

8 Adam LC, Raja J, Ludwig JM, Adeniran A, Gettinger SN, Kim HS. Cryotherapy for nodal metastasis in NSCLC with acquired resistance to immunotherapy. J Immunother Cancer. 2018;6(1):147.

9 Ardavín C, Amigorena S, Reis e Sousa C. Dendritic cells: immunobiology and cancer immunotherapy Immunity. Immunity. 2004 Jan;20(1):17-23

10 McArthur HL, Diab A, Page DB, Yuan J, Solomon SB, Sacchini V, et al. A Pilot Study of Preoperative Single-Dose Ipilimumab and/or Cryoablation in Women with Early-Stage Breast Cancer with Comprehensive Immune Profiling. Clin Cancer Res. 2016;22(23):5729-37.

11 Ross AE, Hurley PJ, Tran PT, Rowe SP, Benzon B, Neal TO, et al. A pilot trial of pembrolizumab plus prostatic cryotherapy for men with newly diagnosed oligometastatic hormone-sensitive prostate cancer. Prostate Cancer Prostatic Dis. 2020;23(1):184-93.

12 Waitz R, Solomon SB, Petre EN, Trumble AE, Fassò M, Norton L, et al. Potent induction of tumor immunity by combining tumor cryoablation with anti-CTLA-4 therapy. Cancer Res. 2012;72(2):430-9.

13 Shaverdian N, Lisberg AE, Bornazyan K, Veruttipong D, Goldman JW, Formenti SC, et al. Previous radiotherapy and the clinical activity and toxicity of pembrolizumab in the treatment of non-small-cell lung cancer: a secondary analysis of the KEYNOTE-001 phase 1 trial. Lancet Oncol. 2017;18(7):895-903.

14 Xing X, Guo J, Ding G, Li B, Dong B, Feng Q, et al. Analysis of PD1, PDL1, PDL2 expression and T cells infiltration in 1014 gastric cancer patients. Oncoimmunology. 2018;7(3):e1356144.

15 Shen X, Zhao B. Efficacy of PD-1 or PD-L1 inhibitors and PD-L1 expression status in cancer: meta-analysis. BMJ. 2018;362:k3529.

16 Wallis CJD, Lawson K, Butaney M, Satkunasivam R, Parikh J, Freedland SJ, et al. Association between PD-L1 status and immune checkpoint inhibitor response in advanced malignancies: a systematic review and metaanalysis of overall survival data. Jpn J Clin Oncol. 2020 Jul 9;50(7):800-809.

17 Griss J, Bauer W, Wagner C, Simon M, Chen M, Grabmeier-Pfistershammer K, et al. B cells sustain inflammation and predict response to immune checkpoint blockade in human melanoma. Nat Commun. 2019;10(1):4186.

18 Cabrita R, Lauss M, Sanna A, Donia M, Skaarup Larsen M, Mitra S, et al. Tertiary lymphoid structures improve immunotherapy and survival in melanoma. Nature. 2020 Jan;577(7791):561-565.

19 Petitprez F, de Reyniès A, Keung EZ, Chen TW, Sun CM, Calderaro J, et al. B cells are associated with survival and immunotherapy response in sarcoma. Nature. 2020 Jan;577(7791):556-560. 